\title{
S-Matrix and Anomaly of de Sitter
}

\author{
Gia Dvali ${ }^{1,2}$
}

1 Arnold Sommerfeld Center, Ludwig-Maximilians-University, 80333 Munich, Germany; gdvali@mpp.mpg.de 2 Max-Planck-Institute for Physics, 80805 Munich, Germany

Citation: Dvali, G. S-Matrix and Anomaly of de Sitter. Symmetry 2021, 13, 3. https://dx.doi.org/10.3390/ sym 13010003

Received: 1 December 2020

Accepted: 17 December 2020

Published: 22 December 2020

Publisher's Note: MDPI stays neutral with regard to jurisdictional claims in published maps and institutional affiliations.

Copyright: () 2020 by the authors. Licensee MDPI, Basel, Switzerland. This article is an open access article distributed under the terms and conditions of the Creative Commons Attribution (CC BY) license (https: / creativecommons.org/ licenses/by/4.0/).

\begin{abstract}
S-matrix formulation of gravity excludes de Sitter vacua. In particular, this is organic to string theory. The $S$-matrix constraint is enforced by an anomalous quantum break-time proportional to the inverse values of gravitational and/or string couplings. Due to this, de Sitter can satisfy the conditions for a valid vacuum only at the expense of trivializing the graviton and closed-string $S$-matrices. At non-zero gravitational and string couplings, de Sitter is deformed by corpuscular $1 / N$ effects, similarly to Witten-Veneziano mechanism in QCD with $N$ colors. In this picture, an $S$-matrix formulation of Einstein gravity, such as string theory, nullifies an outstanding cosmological puzzle. We discuss possible observational signatures which are especially interesting in theories with a large number of particle species. Species can enhance the primordial quantum imprints to potentially observable level even if the standard inflaton fluctuations are negligible.
\end{abstract}

Keywords: S-matrix; de Sitter; quantum gravity

\section{Introduction}

String theory is the most prominent example of a theory based on $S$-matrix. This formulation demands the existence of a valid $S$-matrix vacuum such as Minkowski. It also excludes the de Sitter space-time from the list of the valid vacuum states. The first indication of this appears already at the classical level. It comes from the absence of a globally-defined time in de Sitter space.

Can a de Sitter vacuum exist in quantum theory? Following the authors of [1-3], we give arguments indicating that in a theory with interacting gravitons (closed strings) this is not possible.

In classical General Relativity (GR), the de Sitter metric is sourced by a positive vacuum energy density, $\Lambda$, which we call cosmological constant (or a cosmological term). This parameter is highly sensitive to a cutoff of the theory. This is the essence of the celebrated cosmological constant puzzle. It is usually viewed as the problem of fine-tuning or naturalness. The solution is often attributed to an anthropic selection of our vacuum with small $\Lambda$ on a vast string landscape of plentitude of de Sitter vacua with various energy densities. The arbitrariness accompanying this proposal is sometimes used as the point of criticism as lacking predictivity.

The purpose of the present note is to argue that the situation is exactly the opposite. If there is any quantity that string theory severely constraints, it is $\Lambda$. According to the authors of [1-3], de Sitter vacua are inconsistent with quantum gravity. The problem with positive $\Lambda$ is not a matter of fine tuning but of consistency. The reason is that such "vacua" exhibit an anomalous quantum break-time, $t_{Q}$, incompatible with the notion of a vacuum. The phenomenon of quantum breaking amounts to a full departure from the classical description. Such a departure is evidently in conflict with a stationary (or slow-varying) classical source, such as $\Lambda$. This has number of profound implications. In particular, $\Lambda$ cannot be a source of the energy density in the Universe.

A formulation of quantum gravity in de Sitter space is connected with well-known difficulties (see [4] and references therein). The approach in [1-3,5] changes the perspective on the problem. Instead of treating de Sitter as vacuum, it is viewed as a coherent 
state of gravitons constructed on top of a Minkowski vacuum. This view gives one a double advantage.

First, whatever happens to (or in) de Sitter is now a part of a quantum performance staged on Minkowski. Of course, nothing is free of technicalities, but such a performance can, at least in principle, be traced. Perhaps, the optimal characteristics can be found in terms of what Witten calls meta-observables [4].

The second bonus is that a coherent state description of de Sitter imposes upon us its corpuscular resolution in terms of $N$ constituent gravitons, the picture introduced for de Sitter, as well as for black holes, in [5]. This resolution reveals phenomena that are fundamentally impossible to detect when we treat de Sitter as a vacuum. In particular, the anomalous quantum break-time is caused by $1 / N$ effects [1-3]. The vacuum limit of de Sitter corresponds to $N \rightarrow \infty$, for which the corpuscular effects vanish. In a well-defined sense, these $1 / N$ effects are analogous to the ones in QCD with $N$ colors. There too, $1 / N$ corrections vanish in the 't Hooft's planar limit [6].

The present note is an attempt to substantiate this result from a different angle. We wish to point out that the quantum break-time $t_{O}$ represents a measure of $S$-matrix inconsistency of a de Sitter-like state. The catch is that $t_{Q}$ scales as inverse of the gravitational (or string) coupling. Correspondingly, the parameter choice that supports $t_{Q}=\infty$ decouples gravity.

It is evident that the above link is universal for any $S$-matrix theory of gravity. In particular, it is applicable to string theory, at least at weak string coupling. For any given finite value of the curvature radius, $R_{d S}$, the gravitational quantum break time is proportional to $N$ and inversely proportional to the gravitational coupling $G$. In string theory, $G$ can be expressed through the string scale $M_{S}$ and the string coupling $g_{s}$. Thus, in string theory, for a finite value of the string scale, $M_{s}$, the anomalous quantum break-time scales as,

$$
t_{Q} \propto N R_{d S} \propto \frac{R_{d S}}{g_{s}^{2}},
$$

where the proportionality coefficients are the theory-dependent dimensionless functions of $R_{d S}, M_{S}$ and the number of the light particle species. They are made explicit below.

De Sitter can serve as a valid vacuum only for $t_{Q}=\infty$. From (1), this requires $G=g_{s}=0(N=\infty)$, implying that the closed string and graviton $S$-matrices are trivial. On the other hand, for non-zero values of $g_{s}$ and $G$ (finite $N$ ), the system must exit gracefully from the de Sitter state before the time $t_{Q}$ elapses. This imposes a general constraint that a cosmological source must evolve faster than the quantum break-time.

In this way, thanks to its $S$-matrix formulation, string theory nullifies an outstanding cosmological puzzle. The same must remain true in any effective formulation of Einstein gravity based on $S$-matrix, regardless the nature of UV-completion.

The inconsistency of de Sitter can be described in the language of a quantum anomaly. One may say that the classical symmetry of de Sitter state is explicitly broken by $1 / N$ corpuscular effects. However, to avoid confusion, the meaning of this statement shall be explained very carefully. Usually, the anomaly is associated with the breaking of a symmetry of the Hamiltonian. This breaking may also deform the vacuum. In our S-matrix treatment, the de Sitter is a state on Minkowski [1]. It is mistaken for the "vacuum" by an observer that is blind to $1 / N$ effects. It is the symmetry of this pseudo-vacuum that is broken by $1 / N$ corrections.

In some aspects, this breaking of de Sitter symmetry is spiritually similar to anomalous breaking of axial symmetry by Witten-Veneziano mechanism $[7,8]$ in QCD with $N$ colors. In that case, $1 / N$ effects generate a bias that gives a finite decay-time to a pseudo-Goldstone boson of axial symmetry. Similarly, in case of de Sitter, $1 / N$ effects lead to an anomalous quantum break-time of de Sitter. In both cases, the critical time is determined by N. After this time, de Sitter is no longer able to satisfy a classical equation with the initial source. Thus, to maintain the validity of a classical approximation, necessary for the vacuum treatment, the source must relax towards the asymptotic $S$-matrix vacuum. 
The presented particle physicist's way of thinking is somewhat alien to the way we are used to treat various backgrounds in classical GR. After all, in GR, Minkowski is one out of infinitely many possible choices of the metric. Naively, it is not more special than, let us say, a de Sitter space. Therefore, from the perspective of GR, the de Sitter could be viewed as an equally good vacuum. However, this equality is false. Classically, the Minkowski vacuum represents a limit of de Sitter for $\Lambda \rightarrow 0$. However, from the $S$-matrix perspective, the limit is not smooth. For $\Lambda>0$, no matter how small, a de Sitter state is subjected to a finite anomalous quantum break-time.

\section{Double-Scaling Limit}

As the first indication of discontinuity, we offer the following scaling argument. The argument works in arbitrary number of dimensions but we take $3+1$ first. Let us assume that a fundamental theory of gravity gives us a de Sitter metric sourced by a constant $\Lambda$. The corresponding curvature radius is given by (irrelevant numerical factors shall be ignored from now on),

$$
R_{d S}^{-2}=\Lambda G,
$$

where $G$ is the Newton's gravitational constant.

Let us imagine that we wish to describe some quantum gravitational scattering process on such a vacuum, for example, a $2 \rightarrow 2$ graviton-graviton scattering. Gravitons of some characteristic wavelength $\lambda$ (or effective momentum-transfer $p \sim 1 / \lambda$ ) interact via a quantum gravitational coupling,

$$
\alpha_{g r} \equiv p^{2} G=\frac{G}{\lambda^{2}}
$$

Of course, since there is no global observer, the complete $S$-matrix is impossible. However, we may attempt to replace it by some effective $S$-matrix description. Indeed, if $\lambda \ll R_{d S}$, it appears that the $S$-matrix formulation should be fine, at least approximately. However, this requires the existence of a vacuum that is not affected by the scattered particles. This may appear to be a small detail, but it is crucial for a consistent $S$-matrix description of the process. Putting it differently, the vacuum should not be able to "recoil" and thus absorb some information. In Minkowski, this is guaranteed by an infinite spread and the existence of a global time. The prominent role of Minkowski shall be sharpened later.

To achieve the same in de Sitter, we must pay a price. Namely, we must take the limit of a rigid geometry in which the quantum back-reaction on de Sitter from the scattering particles vanishes. Such a limit is unique:

$$
\Lambda \rightarrow \infty, G \rightarrow 0, \Lambda G=R_{d S}^{-2}=\text { finite }
$$

However, in the very same double-scaling limit, the quantum coupling between the gravitons of any finite wavelength vanishes,

$$
\alpha_{g r}=\frac{G}{\lambda^{2}} \rightarrow 0
$$

Thus, we observe that in the unique limit in which de Sitter can be consistently treated as vacuum, the scattering among gravitons vanishes. In other words, in the vacuum-limit of de Sitter, gravitons decouple:

$$
(\text { deSitter }=\text { vacuum }) \rightarrow \alpha_{g r}=0 .
$$

Notice that, in the same limit, there is no problem of keeping any non-gravitational interaction, e.g., the electroweak interaction, to have a finite strength. Thus, the issue is mainly connected with the quantum gravitational part. This simple scaling argument shows why quantum gravity is special and why it is directly linked with the value of $\Lambda$. 
The above remains true in a full string theory embedding. Gravitons are now the zero modes of the closed strings. The fundamental parameters of the theory are the string scale, $M_{s}$, and a dimensionless string coupling, $g_{s}$. In our discussion, we always stay within the domain of a weak string coupling, $g_{s} \ll 1$.

Let us assume that in string theory we somehow managed to construct a de Sitter space with a curvature radius $R_{d S}$. Intuitively, it is clear that if there exists any sensible notion of de Sitter geometry, it must have a curvature radius $R_{d S}$ larger than the string length $1 / M_{s}$. This intuition is supported by the fact that for $R_{d S} \ll 1 / M_{S}$ the GibbonsHawking temperature [9] of the de Sitter space would exceed the string scale. At such high temperatures, the Hagedorn effects [10] must become important due to an exponentially growing number of active degrees of freedom. Therefore, irrespective of whether the temperatures above $M_{S}$ are reachable in string theory (see, e.g., [11]), it is beyond any reasonable doubt that for such a state the standard geometric description of classical GR must be abolished. Therefore, without loss of generality, we can assume that if de Sitter exists in string theory, its curvature must be below the string scale.

Next, we wish to promote the assumed de Sitter into a quantum vacuum. For this, we must take the double-scaling limit (4). For example, to get a would-be de Sitter vacuum in ten dimensions, we must take the ten-dimensional Newton constant $G_{(10)}$ to zero while keeping $R_{d S}^{-2}=\Lambda G_{(10)}$ finite. However, in such a limit, the string coupling, $g_{s}^{2}=M_{s}^{8} G_{(10)}$, vanishes for any finite value of the string scale $M_{s}$. Thus, as in pure gravity, in full string theory, even a hypothetical existence of de Sitter vacuum is incompatible with the closed string scattering.

The origin of $\Lambda$ in the above example can be made slightly more explicit by invoking a construction in the spirit of [12-17] which uses (anti)D-brane tensions as the source of de Sitter energy. For example, let us, in type IIB theory, pile up $n$ pairs of $D_{9}$-branes and their anti-branes, $\bar{D}_{9}$, on top of one another. The resulting cosmological term $\Lambda$ is given by the sum of the brane tensions,

$$
R_{d S}^{-2}=n g_{s} M_{S}^{2}
$$

As a consistency check, notice that the requirement that de Sitter temperature is less that $M_{S}$ puts a restriction $g_{s} n<1$. This is identical to the constraint on the number of Chan-Paton species [18],

$$
N_{\text {species }}=n^{2}<1 / g_{s}^{2} \text {. }
$$

This expression represents as a special case of the general black hole bound on the number of particle species in a $d$ dimensional theory with a gravitational cutoff scale $M_{*}$ and Newton constant $G_{(d)}[19,20]$

$$
N_{\text {species }}<\frac{1}{M_{*}^{d-2} G_{(d)}} .
$$

For $M_{*}=M_{S}$ in $d=10$, this gives,

$$
N_{\text {species }}<\frac{1}{M_{S}^{8} G_{(10)}}=\frac{1}{g_{s}^{2}} .
$$

The non-gravitational world-volume physics of $D_{9}-\bar{D}_{9}$ is sufficiently well understood [21] and the system is known to be unstable because of the open string tachyon. This instability leads to brane-anti-brane annihilation, which can be described as tachyon condensation, as studied in series of papers (see, e.g., [22]).

Nevertheless, the above brane-anti-brane configuration can produce a classical "hilltop" de Sitter in the limit $g_{s} \rightarrow 0$, while keeping $R_{d S}$ finite. Again, we see that, in the limit in which we can talk about a classically-eternal de Sitter "vacuum", the strings become non-interacting, as do the zero mode gravitons.

The above simple argument encodes a profound point. It shows that a cosmological background can serve as an eternal de Sitter vacuum but only at the expense of trivializing 
quantum gravity. This exposes a fundamental tension between de Sitter vacua and the $S$-matrix description of quantum gravity.

\section{De Sitter as Saturated Coherent State}

A physics common sense should be telling us that there must exists a microscopic reason behind this tension. To identify it, we treat de Sitter as state of gravitons on Minkowski [5]. More precisely, we represent de Sitter as coherent state, $|d S\rangle$, constructed on top of a Minkowski vacuum [1-3]. The choice of a coherent state is dictated by its maximal classicality. Other corpuscular resolutions of de Sitter give similar results [1] (see, [23-27] for various implementations of this proposal).

Now, since Minkowski is a valid $S$-matrix vacuum, this approach seemingly avoids the problem with constant $\Lambda$. For example, the same $2 \rightarrow 2$ graviton scattering process can now be viewed as an $S$-matrix process,

$$
\mathrm{dS}+\operatorname{grav}_{1}+\operatorname{grav}_{2} \rightarrow \mathrm{dS}^{\prime}+\operatorname{grav}_{1}^{\prime}+\operatorname{grav}_{2}^{\prime},
$$

during which the de Sitter coherent state changes, $|\mathrm{d} S\rangle \rightarrow|\mathrm{d} S\rangle^{\prime}$.

This however does not save de Sitter because, even in the absence of any external gravitons, the coherent state $|\mathrm{d} S\rangle$ time-evolves on its own. This evolution leads to a breakdown of classicality after a certain time $t_{Q}$. This phenomenon is referred to as quantum breaking, with the corresponding quantum break-time $t_{Q}$. The time $t_{Q}$ marks a point of a complete departure of the quantum evolution from the classical one. The concept was introduced in [28] and was generalized to de Sitter, inflation and black holes in [1-3]. The outcome of this analysis is that a classical source of a de Sitter type metric must evolve faster than the corresponding $t_{Q}$. In the opposite case, the source cannot be embedded in a quantum theory. In particular, this constraint excludes a constant $\Lambda$. We briefly recount the main ingredients of the story required for the present discussion.

First, viewed as a coherent state, the de Sitter Hubble patch represents a so-called saturated state. Such is a state in which the mean occupation number $(N)$ of dominant constituents and their quantum coupling $(\alpha)$ satisfy the following criticality relation [5],

$$
N=\frac{1}{\alpha}
$$

In the coherent state picture of de Sitter, there exist two types of constituents. The first category originates from the composition of the gravitational field. This category is universal and is present already in pure gravity sourced by a constant $\Lambda$. The second category is represented by non-gravitational quantum constituents of the source, provided the source is dynamical. For example, the role of such a source can be played by an inflaton potential or by a $D$-brane tension, as in [12].

Regardless of the nature of the source, the gravitational constituents have the characteristic frequency $\sim R_{d S}^{-1}$. Their quantum gravitational coupling and the occupation number are related through the saturation relation,

$$
N=\frac{1}{\alpha_{g r}}=\frac{R_{d S}^{2}}{G} .
$$

In general, it is important to understand that the coherent state resolution of de Sitter (or of any other entity) is largely independent of UV-completion. Rather, it amounts to a corpuscular resolution of the state. Such a description probes the IR scales corresponding to the size of the object. This size, in general, is much larger than the cutoff scale. Therefore, if a corpuscular resolution exhibits some inconsistency, this in general cannot be cured by UV-physics. Because of this, a coherent state view of de Sitter "vacuum" is a convenient tool for monitoring its compatibility with the $S$-matrix formulation.

Notice, since, as discussed above, the de Sitter curvature is less than the string scale, the gravitational constituents of the metric are represented by the zero mode sector of 
closed strings. That is, the resolution of the metric is mainly controlled by IR gravitons. The contribution from the closed string states of mass $m$ is exponentially small, $\sim \exp \left(-m R_{d S}\right)$.

Next, we observe that the corpuscular picture gives a well-defined microscopic meaning to the rigid limit (4). In the language of a coherent state, this limit translates as,

$$
N=\frac{1}{\alpha_{g r}} \rightarrow \infty, R_{d S}=\text { finite }
$$

That is, de Sitter remains a saturated state but the number of its constituents becomes infinite. The corpuscular picture allows for a microscopic visualization of how de Sitter is deformed into a valid vacuum in the limit (4) and (14).

To see this, let us consider the previous $2 \rightarrow 2$ scattering process of gravitons on de Sitter background (11). The meaning of the change $|\mathrm{d} S\rangle \rightarrow|\mathrm{d} S\rangle^{\prime}$ is that the inner corpuscular structure of de Sitter is affected. This is due to interactions between the scattered gravitons and the constituents of the de Sitter coherent state. The effect of these interactions can be split in two parts:

(1) A collective recoil. During this process, the recoil momentum is delivered to the entire coherent state, without exciting its individual graviton constituents. This phenomenon can be viewed as something similar to a gravitational Mössbauer effect.

(2) A corpuscular recoil. During this process, the recoil excites some individual quanta of the coherent state. This impact is suppressed by the gravitational coupling (13), and therefore amounts to $1 / N$ effect.

Due to the above two effects, the de Sitter coherent state is altered by the scattering process. However, both back reaction effects vanish in the limit (14). First, the collective recoil is uniformly shared by an infinite number of quanta. Such a state recoils, without being affected. Secondly, the recoil by the individual constituents, since it is suppressed by $1 / N$, also vanishes. This makes it clear why de Sitter becomes a rigid vacuum exclusively in the double-scaling limit (4), or, equivalently, for (14).

This picture also makes transparent the special role of Minkowski as of $S$-matrix vacuum. Indeed, the Minkowski space corresponds to de Sitter with an infinite curvature radius $R_{d S}=\infty$. Correspondingly, viewed as the coherent state of gravitons, Minkowski is composed out of $N=\infty$ gravitons [29]. These gravitons have zero frequencies and infinite wavelengths. Hence, their gravitational couplings $\alpha_{g r}$ vanish even for finite G. Because of this, both recoils vanish. This is why, unlike de Sitter, the Minkowski space is a valid S-matrix vacuum regardless of the value of $G$.

\section{1/N Effects}

We are now approaching a crucial point. Namely, the coherent state resolution of de Sitter likely exposes its intrinsic inconsistency. Due to a perpetual inner re-scattering among its constituents, the de Sitter coherent state loses its classical properties. Note that, for other systems, a departure from classicality is not necessarily an inconsistency. The conflict for de Sitter is that it is sourced by a constant which remains permanently classical.

The potentially-deadly effects are only of order $1 / N$ per Hubble time, but they are persistent and give a dramatic effect over a longer period. It takes time $t_{Q} \sim N R_{d S}(1)$ for the tension to build up. After this time, the "vacuum" evolves into a state that is incompatible with the constant classical source $\Lambda$ that produced de Sitter in the first place. This is an inevitable conflict because the $1 / N$ interactions cannot be switched-off.

As a by-product of the inner re-scattering, the de Sitter coherent state permanently emits particles and depletes. The emitted particles are nothing but the celebrated GibbonsHawking radiation [9]. The corpuscular picture is telling us that the radiation comes from an actual decay of the coherent state [1].

Notice, Gibbons-Hawking radiation is controlled by the collective coupling $\alpha_{g r} N \sim 1$. It therefore survives in the rigid limit (14). In this limit, Gibbons-Hawking spectrum be- 
comes exactly thermal. At the same time, de Sitter becomes an eternal vacuum. The problem, as already explained, is that this limit cannot describe an interacting quantum gravity or strings. A non-trivial quantum gravity requires a finite $N$. Then, the anomalous $1 / N$ corrections give deviations from the thermal spectrum. At the same time, they violate classical de Sitter invariance of the background. These effects vanish for (14) but are present for any finite $N$. In other words, we can say that de Sitter is anomalous with respect to $1 / N$ quantum effects.

To avoid misuse of the language, let us explain better the meaning of the above statement. Usually, anomaly implies a breaking of a classical symmetry by quantum corrections. Once de Sitter is described as a coherent state on Minkowski vacuum, the symmetries of the vacuum are symmetries of Minkowski. The theory of course has a gauge redundancy in form of the general covariance. What is affected by $1 / N$ effects is the symmetry of a coherent state $|d S\rangle=|N\rangle$. For an observer that is blind to $1 / N$ corrections, this coherent state is mistaken for a vacuum. This "vacuum" is invariant under a symmetry that the observer calls a de Sitter group. However, this classical picture is only an approximation that emerges in the limit (14).

To visualize the dynamics, let us oversimplify the story by focusing on a particular decay process. Let the theory contain a set of light particle species, with the masses $\ll 1 / R_{d S}$. Then, an off-shel constituent graviton of energy $\sim 1 / R_{d S}$ can decay into a pair of such particles. Of course, the decay is democratic due to the universality of graviton coupling.

The rate of the process scales as $\Gamma \sim\left(\alpha_{g r} N\right) / R_{d S}$ times the number of species. Thus, the coherent state looses roughly one constituent per time $R_{d s}$. The produced quanta are a part of Gibbons-Hawking radiation which is democratic in species.

As a result of such a decay, the de Sitter coherent state will time-evolve into a (in general entangled) superposition of the sort

$$
|N\rangle \rightarrow \sum_{j} c_{j}|N-1\rangle_{j} \times|j\rangle
$$

where $c_{j}$ are coefficients and index $j$ runs over various states of the produced quanta and the back-reacted de Sitter. Of course, there exist a lot more processes contributing to the evolution and generation of the entanglement but this one suffices to explain the point $($ see $[1,3]$ for more technicalities).

Now, an observer calls the initial state $|N\rangle$ a de Sitter because the expectation value of a graviton field over it gives a classical de Sitter metric. After the decay, this expectation value is going to change in the way that departs from the classical evolution and thus from its classical symmetry. In this sense, a more precise term would be to say that the classical de Sitter symmetry is spoiled rather than broken. The difference is that, in the second case, one should be able to define a time-evolution which, while different from de Sitter, is still classical. Nothing similar to this is feasible because the classicality of the state is affected. That is, $1 / N$ effects break the classical symmetry of the background because they abolish its classicality.

Notice that the above is true, even if we imagine that a symmetry generator, call it $\hat{T}$, that annihilates the state $|N\rangle$, commutes with the time-evolution operator that is responsible for the transition (15). Of course, in such a case $\hat{T}$ also annihilates the right hand side of (15). Thus, the evolved state has the same symmetry as the original one. However, this does not imply that the background remains unchanged. In fact, it is unclear which state should be called a new de Sitter vacuum. It is tempting to say that this is $|N-1\rangle_{j}$, which is obviously different from $|N\rangle$. However, even this choice is not clean, since $|N-1\rangle_{j}$ carries a quantum index through which it is entangled with the rest.

\section{Quantum Break-Time}

In anomalous quantum breaking of de Sitter, its saturation close to criticality (13) is an important factor. While some departure from the classicality is experienced by other macro- 
scopic systems, the saturated ones are special, as they can depart fully. Without repeating technicalities that can be found in $[1-3,28]$, below we display some useful equations.

For a generic saturated system, in the absence of a classical Lyapunov instability, the quantum break-time is given by the following formula [3],

$$
t_{Q}=\frac{t_{\mathrm{cl}}}{\alpha N_{\text {species }}}=t_{\mathrm{cl}} \frac{N}{N_{\text {species }}},
$$

where $\alpha$ is the quantum coupling and $t_{\mathrm{cl}}$ is a characteristic time on which classical nonlinearities become important. As introduced above, $N_{\text {species }}$ counts the number of the active light particle species in the spectrum of the theory that interact via coupling $\alpha$. This number should not be confused with the occupation number of quanta $N$.

Notice that, generically, the point $\alpha N_{\text {species }}=1$ marks a non-perturbative saturation of unitarity by the species [30]. In this respect, the bound (9) represents a particular manifestation of this phenomenon in case of gravity. Thus, Equation (16) tells us that, in a unitary system, the quantum break-time $t_{Q}$ cannot be shorter than the classical time $t_{\mathrm{cl}}$.

Now, the systems that are subject to classical instabilities are special from quantum breaking point of view. If a system exhibits a classical Lyapunov time $t_{\mathrm{cl}}=t_{\text {Lyapunov }}$, the quantum break-time can be as short as [28],

$$
t_{Q}=t_{\text {Lyapunov }} \ln \left(\alpha^{-1}\right)=t_{\text {Lyapunov }} \ln (N) .
$$

Thus, a classical instability can speed up the process of quantum breaking. However, in a generic system, the quantum break-time is not necessarily connected with instability but always signals a breakdown of the classical description.

\section{S-Matrix and Quantum Breaking}

Using a proper expression for $t_{Q}$, it is easy to follow how the $S$-matrix constraint goes hand in hand with the quantum break-time criterion. For example, in $d=4$ theory of pure gravity, we must take $t_{\mathrm{cl}}=R_{d S}$ and $\alpha=\alpha_{g r}=G / R_{d S}^{2}$. Then, from (16), the resulting quantum break-time is [1-3],

$$
t_{Q}=\frac{R_{d S}^{3}}{G} .
$$

The de Sitter vacuum is only possible if this time is taken infinite. This requires, $G \rightarrow 0$. However, the gravitational $S$-matrix then becomes trivial.

The existence of additional particle species changes the story. According to (16), the quantum break-time is shortened if the theory contains a large number $N_{\text {species }}$ of light particle species. In this case, Equation (18) must be replaced by,

$$
t_{Q}=\frac{R_{d S}^{3}}{G N_{\text {species }}} .
$$

Taking into account the black hole bound (9) on $N_{\text {species, }}$ we can convert the above equation into the following bound,

$$
t_{Q}>R_{d S}\left(R_{d S} M_{*}\right)^{2} .
$$

This expression tells us that, as long as $R_{d S}$ is larger than the cutoff length $1 / M_{*}$, the quantum break-time of de Sitter is longer than the Hubble time.

The existence of particle species has important effect on gravitational physics, both via black holes and via cosmology. This is already clear from the fact $[19,20]$ that species lower the gravitational cutoff of the theory $M_{*}$ (relative to Planck mass $\equiv 1 / G_{(d)}^{1 /(d-2)}$ ) according

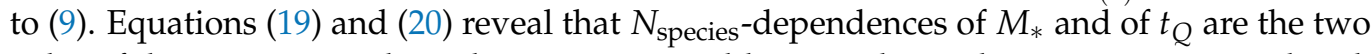
sides of the same coin. This is because no sensible semi-classical state can quantum break during the time $t_{Q}$ shorter than (or comparable to) its classical time $t_{\mathrm{cl}}$. As is clear from (16), the increase of the number of species speeds up the quantum breaking process and moves 
$t_{Q}$ closer to $t_{\mathrm{cl}}$. A system for which $t_{Q} \sim t_{\mathrm{cl}}$ cannot be described within a unitary effective theory. For such a system, $t_{Q}$ marks the cutoff length.

To highlight the important role of species, let us take another double-scaling limit,

$$
G \rightarrow 0, N_{\text {species }} \rightarrow \infty, G N_{\text {species }}=\text { finite, } R_{d S}=\text { finite } .
$$

To keep $R_{d S}$ finite, this must be combined with the previous large- $N$ limit (14) (or, equivalently, with (4)). The limit (21) is also analogous to 't Hooft's planar limit in QCD. The role of the number of colors in gravity is assumed by the total number of particle species $N_{\text {species }}$.

One interesting thing about the above limit is that, despite the fact that the gravitational coupling vanishes, the quantum break-time (19) remains finite. This is due to the collective effect of species. Thus, in the combined limit (21), de Sitter never becomes a rigid vacuum.

This phenomenon can be understood in two languages. First, since $R_{d S}$ is finite, one naively expects to have a constant Gibbons-Hawking temperature $T_{G H}=1 / R_{d S}$. However, this is not possible since, under the assumption of an exact thermality, the intensity of Gibbons-Hawking radiation would diverge due to infinite number of light species. Thus, the back reaction remains non-zero and must be taken into account.

The second way to understand the same effect is from the point of view of the coherent state picture of de Sitter [1-3]. The radiation is a result of the depletion of the coherent state. This is due to decays and various re-scatterings of its constituents. For example, the simplest processes are the direct decays of constituent off-shell gravitons, or their pairwise annihilations into species. As already noted, due to universality of the gravitational interaction, the particle creation is democratic in species. This explains the universality of Gibbons-Hawking radiation. The resulting half-decay time of the $\mathrm{N}$-graviton coherent state is

$$
t_{\text {decay }}=R_{d S} \frac{N}{N_{\text {species }}} .
$$

Taking into account (13), this expression exactly matches (19).

Thus far, we did not specify the internal dynamics of the energy density source that produces the de Sitter state. Of course, for constant $\Lambda$, there is no dynamics and the entire quantum breaking process is due to gravity. The finiteness of $t_{Q}$ reveals the inconsistency of $\Lambda$. Thus, a valid source must change in time, in order to avoid a conflict with quantum break-time. Such a source, is expected to have its internal non-gravitational dynamics. An example is a scalar field with a potential that asymptotes to zero.

Here, we must distinguish among the two types of processes that contribute to quantum breaking: (1) re-scattering of the constituent gravitons; and (2) re-scattering of the constituents of the source. The first mechanism is generic and is independent of the composition of the source. It therefore provides a universal constraint.

However, the quantum breaking effect can independently be exhibited by a nongravitational dynamics of the source. The corresponding quantum break-time is expected to obey (16), where the parameters $\alpha, N, t_{\mathrm{cl}}$ must be understood as the non-gravitational characteristics of the source. For example, $N$ is the occupation number of a scalar field in the coherent state, $\alpha$ is its self-coupling, and $t_{\mathrm{cl}}$ is the corresponding classical time (see $[1,3,28,31-34]$ for applications of quantum breaking to some inflationary scenarios and other systems.)

If in a given theory, a potential energy of a scalar field can decrease faster than the quantum break-time of the combined system (gravity plus a scalar), such evolution can be well-described by a classical slow-roll. In this situation, de Sitter can asymptote to Minkowski vacuum without ever encountering a full quantum breaking. In quantum language, such a classical evolution corresponds to an evolution of two coupled coherent states (scalar and gravity), during which the quanta evolve collectively. That is, the evolution is governed by the collective effects that have a strength set by $\alpha N$. At the same time, the recoils of individual quanta ( $1 / N$ effects) are un-efficient, since they have no time to influence the system.

During this evolution, the decrease of the scalar potential energy causes the increase of the de Sitter Hubble radius $R_{d s}$. Thus, the wavelengths of the gravitons increase. 
Correspondingly, the occupation number $N$ increases according to (13). Due to this, the system never catches up with its quantum break-time (18), which is pushed further and further due to the growth of $R_{d s}$. The end point of such an evolution is the Minkowski vacuum, which corresponds to a coherent state with $N=\infty, R_{d S}=\infty$.

\section{7. $t_{Q}$ in String Theory}

Let us now move to string theory. Let us assume that a would-be de Sitter of curvature radius $R_{d S}$ is achieved by some construction. Since $R_{d S}$ sets the wavelengths/frequencies of graviton constituents of the de Sitter coherent state, their quantum coupling is given by,

$$
\alpha_{g r}=\frac{G_{(10)}}{R_{d S}^{8}}=\frac{g_{s}^{2}}{\left(R_{d S} M_{s}\right)^{8}} .
$$

Plugging this into (16) and taking into account $t_{\mathrm{cl}}=R_{d S}$, we obtain the following expression for quantum break-time,

$$
t_{Q}=R_{d S} \frac{\left(R_{d S} M_{S}\right)^{8}}{g_{s}^{2}} \frac{1}{N_{\text {species }}} .
$$

Here, $N_{\text {species }}$ is the number of light particle species with masses below $1 / R_{d S}$. An example is given below. It is obvious that, for finite values of $R_{d S}$ and $M_{S}$, the only way of making $t_{Q}$ infinite is by taking $g_{s}=0$. This renders the closed string $S$-matrix trivial.

Since the expressions (23) and (24) make no assumptions about the nature of the source, they set a universal upper bound on the quantum break-time. This upper bound is sufficient for excluding de Sitter vacua from string theory. However, in particular cases, especially when the source exhibits a Lyapunov exponent, the quantum breaking of the source itself could happen much faster. Of course, such an instability only adds to the tension against de Sitter. However, the gravitational contribution (24) to $t_{Q}$, persists regardless of other effects.

For example, let us come back to the system with $n$ pairs of $D_{(9)}-\bar{D}_{(9)}$-branes piled up on top of each other. In this case, the curvature radius is given by (7). From (23), the corresponding quantum coupling of the graviton constituents of de Sitter is,

$$
\alpha_{g r}=\left(n g_{s}\right)^{4} g_{s}^{2} .
$$

Taking into account the number of Chan-Paton species, $N_{\text {species }}=n^{2}$, and using (16), we get the following quantum break-time,

$$
t_{Q}=\frac{1}{M_{s}} \frac{1}{\left(n g_{s}\right)^{13 / 2}}=\frac{R_{d S}}{\left(n g_{s}\right)^{6}} .
$$

As another consistency check, notice a quartic agreement. First, the requirement that the de Sitter quantum break-time is less than the Hubble time, $t_{Q}<R_{d S}$, puts the bound on the number of Chan-Paton factors, given by (8). This is exactly the same bound as was derived in [18] by imposing the black hole bound [19] (9) on Chan-Paton species. Next, as noticed above, the same bound comes from the requirement that de Sitter temperature is less than the string scale. Finally, according to the arguments presented in [30], the same bound is imposed by the unitarity of Chan-Paton scattering amplitudes.

Now, the quantum break-time due to instability of the source is much shorter. Indeed, taking into account the Lyapunov exponent originating from the open string tachyon $t_{\text {Lyapunov }} \sim M_{S}^{-1}$ and using (17), we get,

$$
t_{Q}=\frac{1}{M_{s}} \ln \left(g_{s}^{-2}\right) \text {. }
$$


This type of quantum breaking can in principle be avoided by assuming that the $D$-brane system somehow got stabilized in a local minimum. Of course, this would kill the Lyapunov exponent and eliminate (27). We are ready to grant such a possibility.

However, regardless of this assumption, there is no visible way to eliminate (24), as it comes from the gravitational re-scattering of finite anergy quanta. Therefore, this expression sets an upper bound on quantum break-time of an arbitrary de Sitter-like state in string theory.

\section{Connection to Witten-Veneziano}

In the picture presented in [1-3], which we follow, de Sitter is not a vacuum but rather a coherent state built on top of Minkowski. Thus, the de Sitter symmetry is not a property of a vacuum but an effective (emergent) symmetry of a particular coherent state with $N$ constituents. This symmetry becomes exact in the limit of infinite $N(14)$ which is equivalent to (4). For a finite $N$, it is abolished by $1 / N$ quantum corrections.

The anomalous decay of de Sitter due to $1 / N$ effects cries for establishing analogy with the breaking of axial symmetry by Witten-Veneziano mechanism in QCD with $N$-colors $[7,8]$. Without any reference to fundamental fermions, this theory can be viewed as theory of mesons and glueballs. They interact via a quantum coupling that has $1 / N$ strength. The theory also includes baryons with mass $\sim N$. As in the corpuscular picture of de Sitter, the classical limit corresponds to $N=\infty$. In this limit, there exists an exact shift symmetry of one of the mesons $\left(\eta^{\prime}\right)$, a Goldstone bosons corresponding to a non-linearly realized axial $U(1)$. However, at the quantum level, this symmetry is explicitly broken by $1 / N$ effects which generate mass and a finite lifetime for the $\eta^{\prime}$-meson. In the certain sense, this is similar to how the quantum $1 / N$ effects generate a finite quantum break-time for de Sitter. However, unlike the $\eta^{\prime}$-meson, the quantum breaking of de Sitter appears to be in conflict with its classical source, unless the source evolves in time.

For establishing a closer connection, the analogy must be drawn among the wouldbe vacuum states in two theories. On cosmology side, such is a de Sitter state. The corresponding state on the QCD side, is represented by a pseudo-Goldstone $\eta^{\prime}$ field displaced away from its true vacuum. In quantum theory, such a field is described as a coherent state of $\eta^{\prime}$ bosons. With this identification, the close parallels, both for finite and infinite $N$, become more transparent.

As explained above, at finite $N$, de Sitter cannot describe a true vacuum. Instead, it is a coherent state that undergoes a quantum breaking after a finite time $t_{Q}$. Likewise, in QCD, a pseudo-Goldstone $\eta^{\prime}$ field, displaced away from the true minimum, is not a valid vacuum state. Rather, due to a non-zero mass, it will start to relax towards the true vacuum and will perform the decaying coherent oscillations about it.

Similarly to de Sitter, such an oscillating scalar field, represents a coherent state. In this case, as said, the constituents are $\eta^{\prime}$ pseudo-Goldstone bosons. Thus, the proposed analogy, maps the coherent state of gravitons in de Sitter cosmology, on a coherent state of $\eta^{\prime}$ mesons in QCD. In this connection, the Hubble radius of de Sitter, $R_{d S}$, plays the role analogous to the QCD length. Not surprisingly, the Planck mass is mapped on the decay constant of the $\eta^{\prime}$ meson.

Analogously, for $N=\infty$, the situations in the two theories are very similar. At infinite $N$, de Sitter represents a valid (eternal) vacuum, since the quantum break-time becomes infinite. Likewise, in QCD at $N=\infty$, the curvature of the Goldstone potential vanishes. Correspondingly, the relaxation time becomes infinite. In this situation, a displaced (pseudo)Goldstone field, with zero initial velocity, stays eternally in a displaced position.

At the end of the day, the above similarities need not be taken more than an analogy. Nevertheless, the close connection of the two physical pictures is rather striking. The underlying reason is in certain universal aspects of large- $N$ physics. 


\section{Some Comparisons}

It is useful to comment on how the conjectures by other authors fit within the presented framework. We start with the one by Banks [35]. While this work contains several points on which we have no immediate baring, one sharp conjecture made there is that de Sitter Hilbert space has a finite dimensionality. This statement can be taken with some skepticism. However, the present picture offers an interpretation and possibly some support.

As discussed above, our view is that in an S-matrix theory of gravity de Sitter cannot be a vacuum. Instead, it must be viewed as a composite [5] coherent state in the Hilbert space built on top of the $S$-matrix vacuum of Minkowski [1-3]. Of course, the entire Hilbert space is infinite with de Sitter being a particular composite state in it. Nevertheless, to the notion of finiteness of the "de Sitter's Hilbert space" can be given a well defined meaning. We can attribute it to a dimensionality of the portion of the Hilbert space explored by the state vector over the quantum break-time $t_{Q}$. This is a meaningful prescription, since beyond $t_{Q}$ the state vector does not describe anything close to a classical de Sitter vacuum.

Several authors (see, e.g., Tsamis and Woodard [36], Polyakov [37], and Anderson, Mottola, and Sanders [38]) have suggested instabilities of de Sitter space. These proposals are very different (both from us and also from each other) but touch a certain common point. We share the point (especially with the approach by Polyakov) that de Sitter cannot keep producing particles "for free" and some price must be payed. The difference is that in these papers it is envisaged that, due to back reaction, either de Sitter is destabilized or the curvature of de Sitter space is decreasing in time. Basically, effectively $\Lambda$ is getting screened as the time goes on. This is not necessarily in contradiction to our findings but represents a fundamentally different claim.

The picture [1-3] that we advocate is not of a decrease of the classical curvature (or screening $\Lambda$ ) in time. It is in principle impossible to describe the quantum evolution of the de Sitter coherent state, triggered by $1 / N$ effects, in terms of time-dependent classical characteristics such as the curvature. Rather, $1 / N$ effects induce a departure from the classical description and its complete invalidation after the time $t_{Q}$. Therefore, in our picture, the de Sitter with a constant $\Lambda$ is inconsistent rather than unstable.

Of course, if different quantum gravity effects happen to screen $\Lambda$ on times shorter than $t_{Q}$, this may help in avoiding the inconsistency. A priori, we have nothing against such a possibility but it is beyond our claims.

Finally, some constraints on the scalar potentials that speak against de Sitter were also conjectured recently [39,40]. As discussed in [41,42], these constraints are essentially equivalent to the ones obtained from the quantum break-time [1-3], for a special choice of $\alpha \sim 1$.

\section{Observational Signatures and Power of Species}

We briefly outline some observational signatures that follow from our approach. Since our work is a continuation of the framework in [1-3], part of the signatures have already been discussed there. For completeness, we outline the main directions and then discuss some new ideas.

The first obvious prediction, relevant for the present day cosmology, is the exclusion of a constant $\Lambda$ from the energy density budget in the Universe. In this light, more precision tests of standard $\Lambda \mathrm{CDM}$ cosmology [43] are called for. Our picture indicates that whatever contributes to the accelerated expansion of the Universe, cannot be $\Lambda$, or any other constant source. We do not see how such a source could be reconciled with the finite quantum break-time.

Next, a more indirect contact with observations can be established though the quantum breaking constraints imposed on inflaton potentials. The requirement that a scalar field must evolve faster than the corresponding $t_{Q}(16)$, puts a constraint on the shape of its 
potential [1]. In a large class of theories, this effectively translates as an upper bound on the inflationary slow-roll parameters which limits the number of inflationary e-folds by,

$$
\mathcal{N}_{e} \lesssim \frac{1}{\alpha N_{\text {species }}}
$$

In particular, applied to string theory, we must take $\alpha=g_{s}^{2}$.

Perhaps, the most interesting qualitatively-new observables are the corpuscular imprints from the inflationary epoch [1,44]. The essence of the story is as follows. The inflationary paradigm is based on the assumption that the present Hubble patch underwent through a de Sitter epoch. In the semi-classical treatment, there is no upper bound on the duration of this phase. At the same time, the observable imprints are assumed to come only from last 60 or so e-folds. In the semi-classical picture, the entire information about the prior epoch is lost.

This is fundamentally changed in the corpuscular picture [1], which is imposed upon us by the $S$-matrix consistency. This picture tells us that de Sitter possesses an intrinsic quantum clock. This clock is powered by $1 / N$ corpuscular effects. After the time $t_{Q}$, this leads to a complete quantum breakdown of the coherent state. Basically, one can say that de Sitter "wears off".

By consistency, we know that our Hubble patch gracefully exited the de Sitter phase well before $t_{Q}$ elapsed. That is, the exit time, $t_{\text {exit }}=\mathcal{N}_{e} R_{d S}$, that measures the classical duration of inflation, must satisfy,

$$
t_{\text {exit }} \lesssim t_{Q}
$$

Now, since the strength of the corpuscular imprints is $\sim R_{d S} / t_{Q}$ per Hubble time, by the end of inflation, the relative magnitude of the imprints is given by [1],

$$
\delta=\frac{t_{\text {exit }}}{t_{Q}} .
$$

Thus, the longer does the inflation last, the stronger are the quantum imprints and the higher are the chances of detecting them via the precision cosmology.

A successful inflation must last longer than 60 e-folds to solve the horizon and flatness problems. Taking this into account, we can write a combined bound,

$$
\frac{60 R_{d S}}{t_{Q}}<\delta<\epsilon_{\mathrm{obs}}
$$

where $\epsilon_{\text {obs }}$ parameterizes the current observational accuracy.

This brings us to the following point. The existence of a large number of particle species can dramatically enhance the observable effect of quantum imprints. This is revealed by the equation (19), which is telling us that species shorten $t_{Q}$. Using the expression (19), we can convert (31) into a useful bound on the number of inflationary efolds,

$$
60<\mathcal{N}_{e}<\frac{N}{N_{\text {species }}} \epsilon_{\text {obs }}=\frac{1}{N_{\text {species }}} \frac{R_{d S}^{2}}{G} \epsilon_{\text {obs }},
$$

where in the last equality we expressed $N$ through (13). At the same time, the relative strength of imprints (30) takes the form,

$$
\delta \sim \mathcal{N}_{e} \frac{N_{\text {species }}}{N} .
$$

We see that the number of species enhances the amplitude and simultaneously narrows the window (32).

In general, a scenario with $\frac{N_{\text {species }}}{N} \sim \frac{\epsilon_{\text {obs }}}{60}$, puts the effect near the observational accuracy. This fact opens a large range of inflationary scenarios, with different values of 
Hubble, for which the effect can be observable. Notice that already taking into account the existing number of particle species in the Standard Model increases the effect by a factor of a hundred, as compared to the case with pure inflaton.

Of course, the best motivated are the theories in which the existence of the large number of species is independently justified. Many extension of the Standard Model (e.g., grand unification), have this property. We wish to discuss an extreme case. This is a scenario with $N_{\text {species }} \sim 10^{32}$ particle species, which is motivated by the Hierarchy Problem [19]. This number represents an absolute phenomenological upper bound, since through (9) it brings the cutoff down to $M_{*} \sim \mathrm{TeV}$. Correspondingly, the Hierarchy Problem gets nullified. In a theory with such a large number of particle species, the amplitude of corpuscular imprints can easily reach the observable accuracy for the values of Hubble parameter as low as $R_{d S}^{-1} \sim 10 \mathrm{GeV}$.

In general, theories with many species open up a qualitatively new way of generating observable quantum effects for the values of the Hubble parameter for which the standard inflationary fluctuations would be undetectable. The species enhance the effect on two fronts.

First, their production due to decay of the coherent state increases the relative energy density of Gibbons-Hawking radiation as compared to the energy of the inflaton background,

$$
\delta_{s p}=\frac{N_{\text {species }}}{N}=N_{\text {species }} \frac{G}{R_{d S}^{2}} .
$$

Secondly, due to back reaction on the de Sitter coherent state, the species imprint the corpuscular corrections with the strength given by (33).

In today's observations, the imprint (34) can be detectable only from the species that were emitted from the coherent state during the last 60 e-folds. In contrast, the imprint of back reaction (34) is cumulative due to ongoing depletion since the onset of inflation. This imprint carries a quantum information about the entire duration of inflation. It is remarkable how the $S$-matrix consistency correlates the two contributions.

It is interesting that, for large values of $N$ and $N_{\text {species, }}$ the contributions from species can dominate over the standard contribution from the fluctuations of the inflaton field. The reason is that, although at large $N$ (small Hubble) all contributions are suppressed, the imprints from the corpuscular effects, (33) and (34), are enhanced by the number of species $N_{\text {species, }}$ whereas the standard inflaton fluctuations are not. This can also be understood from the fact that in the limit

$$
N \rightarrow \infty, \frac{N_{\text {species }}}{N}=\text { finite }
$$

the only surviving coupling is the collective coupling of species. All other interactions decouple. Notice that the limit (35) is equivalent to (21), in which case gravity decouples while $t_{Q}$ stays finite. The imprints from species encode the information about the finiteness of $t_{Q}$. In such a regime, the fluctuations enhanced by species can be a dominant source of primordial quantum imprints in the Universe. We thus observe that the theories with species can offer a possibility of probing the quantum sub-structure of the inflationary density perturbations in a qualitatively different way.

\section{Outlook}

It is evident [1-3] that de Sitter vacua are inconsistent in quantum theory. In this paper, we reconcile this view with the $S$-matrix perspective. We observe how the quantum breaking of de Sitter vacua is linked with their conflict with $S$-matrix. This conflict is due to $1 / N$ effects that generate a departure from classicality. A full quantum break takes place after a finite time $t_{Q}$. These effects vanish only in the limit of infinite $N$. However, this limit implies $G=0$ and, in string theory, $g_{s}=0$. 
We pay special attention to the applications and cross-checks in string theory, as a prominent example of $S$-matrix theory. However, the presented arguments are very general and should be applicable to an arbitrary $S$-matrix formulation of Einstein gravity, regardless of the UV-completion. In particular, they are applicable to UV-completion by classicalization $[45,46]$

We converge to the following statement. For fixed $R_{d S}$ and $M_{s}$, the quantum breaking time of stringy de Sitter scales with $g_{s}$ as (1). It is unclear how to reconcile such a state with a classical source that last longer than $t_{Q}$. In such a case, a de Sitter-like state in string theory, irrespective of a seeming classical stability of the source, can be neither stable nor meta-stable. The only possibility for obtaining an eternal de Sitter, is to make the quantum break-time infinite (while keeping $R_{d S}$ finite). This demands $g_{s} \rightarrow 0$, in which case the string $S$-matrix becomes trivial.

In general, a remedy for a de Sitter state is to asymptote to Minkowski vacuum sufficiently fast. The term sufficiently fast means faster than its local quantum break-time, $t_{Q}$. In other words, for $S$-matrix consistency, the graceful exit time from de Sitter, $t_{\text {exit, }}$ must be shorter than the quantum break-time, (29).

Naturally, the above puts severe constraints on de Sitter model building. Based on the idea that $D$-branes can be used for creating de Sitter [12], a considerable effort went in engineering such states in string theory. The presented $S$-matrix argument tells us that any such configuration must evolve on the time-scales shorter than the corresponding $t_{Q}$.

As discussed above [1-3], the quantum break-time constraint has a number of consequences, including the observational ones. First, of course, it excludes a constant $\Lambda$ as part of the energy density in the Universe. Whatever source contributes into the dark energy must be time-dependent.

Secondly, the presented picture point to new types of imprints from the inflationary epoch $[1,44]$. These are the imprints from $1 / N$ corpuscular effects that power the quantum break-time clock. The strength of these imprints is set by a parameter $\delta=t_{\text {exit }} / t_{Q}$, also given by (33). Correspondingly, the inflationary scenarios with short $t_{Q}$ are the most interesting ones.

The number of particle species, $N_{\text {species, }}$ plays a special role in shortening $t_{Q}$ and generating observable imprints from the inflationary epoch. Remarkably, through the quantum breaking constraint, the imprints that come from last 60 e-folds (34) are linked with the ones that originate from the onset of inflation (33). The latter imprints carry memories of the entire duration of inflation.

An important point is that the strengths of the corpuscular imprints, (33) and (34), are enhanced by $N_{\text {species, }}$ while the standard inflaton fluctuations, are not. Due to this, species can provide a way of generating the observable imprints even for the values of the Hubble parameter for which the standard inflaton fluctuations are negligible. Therefore, in a wide range of the inflationary models, the corpuscular effects, enhanced by the number of species, can provide a major observational window in the quantum substructure of the inflationary de Sitter.

Funding: This research received no external funding.

Acknowledgments: We thank Goran Senjanović for valuable discussions and comments. Conversations on de Sitter N-portrait with Lasha Berezhiani, Cesar Gomez and Sebastian Zell are also acknowledged. This work was supported in part by the Humboldt Foundation under Humboldt Professorship Award, by the Deutsche Forschungsgemeinschaft (DFG, German Research Foundation) under Germany's Excellence Strategy-EXC-2111—390814868, and Germany's Excellence Strategy under Excellence Cluster Origins.

Conflicts of Interest: The author declares no conflict of interest. 


\section{References}

1. Dvali, G.; Gomez, C. Quantum Compositeness of Gravity: Black Holes, AdS and Inflation. J. Cosmol. Astropart. Phys. $2014,1,023$. [CrossRef]

2. Dvali, G.; Gomez, C. Quantum Exclusion of Positive Cosmological Constant? Annalen Phys. 2016, 528, 68-73. [CrossRef]

3. Dvali, G.; Gomez, C.; Zell, S. Quantum Break-Time of de Sitter. J. Cosmol. Astropart. Phys. 2017, 06, 028. [CrossRef]

4. Witten, E. Quantum gravity in de Sitter space. arXiv 2001, arXiv:hep-th/0106109.

5. Dvali, G.; Gomez, C. Black Hole's Quantum N-Portrait. Fortsch. Phys. 2013, 61, 742-767. [CrossRef]

6. Hooft, G. A Planar Diagram Theory for Strong Interactions. Nucl. Phys. B 1974, 72, 461. [CrossRef]

7. Witten, E. Current Algebra Theorems for the U(1) Goldstone Boson. Nucl. Phys. B 1979, 156, 269-283. [CrossRef]

8. Veneziano, G. U(1) Without Instantons. Nucl. Phys. B 1979, 159, 213-224. [CrossRef]

9. Gibbons, G.W.; Hawking, S.W. Cosmological Event Horizons, Thermodynamics, and Particle Creation. Phys. Rev. D 1977, 15, 2738-2751. [CrossRef]

10. Hagedorn, R. Statistical thermodynamics of strong interactions at high-energies. Nuovo Cim. Suppl. 1965, 3, 147-186.

11. Bowick, M.J. Finite temperature strings. arXiv 1992, arXiv:hep-th/9210016.

12. Dvali, G.R.; Tye, S.H.H. Brane inflation. Phys. Lett. B 1999, 450, 72-82. [CrossRef]

13. Dvali, G.R. Infrared hierarchy, thermal brane inflation and superstrings as superheavy dark matter. Phys. Lett. B 1999, 459, 489-496. [CrossRef]

14. Dvali, G.R.; Shafi, Q.; Solganik, S. D-brane inflation. arXiv 2001, arXiv:hep-th/0105203.

15. Burgess, C.P.; Majumdar, M.; Nolte, D.; Quevedo, F.; Rajesh, G.; Zhang, R.J. The Inflationary brane anti-brane universe. J. High Energy Phys. 2001, 07, 047. [CrossRef]

16. Kachru, S.; Kallosh, R.; Linde, A.D.; Trivedi, S.P. De Sitter vacua in string theory. Phys. Rev. D 2003, 68, 046005. [CrossRef]

17. Kachru, S.; Kallosh, R.; Linde, A.D.; Maldacena, J.M.; McAllister, L.P.; Trivedi, S.P. Towards inflation in string theory. J. Cosmol. Astropart. Phys. 2003, 10, 013. [CrossRef]

18. Dvali, G.; Gomez, C. Species and Strings. arXiv 2010, arXiv:1004.3744.

19. Dvali, G. Black Holes and Large N Species Solution to the Hierarchy Problem. Fortsch. Phys. 2010, 58, 528-536. [CrossRef]

20. Dvali, G.; Redi, M. Black Hole Bound on the Number of Species and Quantum Gravity at LHC. Phys. Rev. D 2008, 77, 045027. [CrossRef]

21. Srednicki, M. IIB or not IIB. J. High Energy Phys. 1998, 8, 5. [CrossRef]

22. Sen, A. NonBPS states and Branes in string theory. arXiv 1999, arXiv:hep-th/9904207.

23. Kühnel, F. Thoughts on the Vacuum Energy in the Quantum N-Portrait. Mod. Phys. Lett. A 2015, 30, 1550197. [CrossRef]

24. Kuhnel, F.; Sandstad, M. Corpuscular Consideration of Eternal Inflation. Eur. Phys. J. C 2015, 75, 505. [CrossRef]

25. Casadio, R.; Kuhnel, F.; Orlandi, A. Consistent Cosmic Microwave Background Spectra from Quantum Depletion. J. Cosmol. Astropart. Phys. 2015, 9, 2. [CrossRef]

26. Berezhiani, L. On Corpuscular Theory of Inflation. Eur. Phys. J. C 2017, 77, 106. [CrossRef]

27. Brahma, S.; Dasgupta, K.; Tatar, R. Four-dimensional de Sitter space is a Glauber-Sudarshan state in string theory. arXiv 2020, arXiv:2007.00786.

28. Dvali, G.; Flassig, D.; Gomez, C.; Pritzel, A.; Wintergerst, N. Scrambling in the Black Hole Portrait. Phys. Rev. D 2013, 88, 124041. [CrossRef]

29. Dvali, G.; Gomez, C.; Lüst, D. Classical Limit of Black Hole Quantum N-Portrait and BMS Symmetry. Phys. Lett. B 2016, 753, 173-177. [CrossRef]

30. Dvali, G. Entropy Bound and Unitarity of Scattering Amplitudes. arXiv 2020, arXiv:2003.05546.

31. Dvali, G.; Zell, S. Classicality and Quantum Break-Time for Cosmic Axions. J. Cosmol. Astropart. Phys. 2018, 07, 064. [CrossRef]

32. Kovtun, A.; Zantedeschi, M. Breaking BEC. J. High Energy Phys. 2020, 07, 212. [CrossRef]

33. Kovtun, A.; Zantedeschi, M. Breaking BEC: The fast and the quantum. arXiv 2020, arXiv:2008.02187.

34. Berezhiani, L.; Zantedeschi, M. On Evolution of Coherent States as Quantum Counterpart of Classical Dynamics. arXiv 2020, arXiv:2011.11229.

35. Banks, T. Cosmological breaking of supersymmetry? Int. J. Mod. Phys. A 2001, 16, 910-921. [CrossRef]

36. Tsamis, N.C.; Woodard, R.P. Quantum gravity slows inflation. Nucl. Phys. B 1996, 474, 235-248. [CrossRef]

37. Polyakov, A.M. Infrared instability of the de Sitter space. arXiv 2012, arXiv:1209.4135.

38. Anderson, P.R.; Mottola, E.; Sanders, D.H. Decay of the de Sitter Vacuum. Phys. Rev. D 2018, 97, 065016. [CrossRef]

39. Obied, G.; Ooguri, H.; Spodyneiko, L.; Vafa, C. De Sitter Space and the Swampland. arXiv 2018, arXiv:1806.08362.

40. Ooguri, H.; Palti, E.; Shiu, G.; Vafa, C. Distance and de Sitter Conjectures on the Swampland. Phys. Lett. B 2019, 788, 180-184. [CrossRef]

41. Dvali, G.; Gomez, C. On Exclusion of Positive Cosmological Constant. Fortsch. Phys. 2019, 67, 1800092. [CrossRef]

42. Dvali, G.; Gomez, C.; Zell, S. Quantum Breaking Bound on de Sitter and Swampland. Fortsch. Phys. 2019, 67, 1800094. [CrossRef]

43. Secrest, N.J.; von Hausegger, S.; Rameez, M.; Mohayaee, R.; Sarkar, S.; Colin, J. A Test of the Cosmological Principle with Quasars. 4 citations counted in INSPIRE as of 25 Nov 2020. arXiv 2020, arXiv:2009.14826.

44. Dvali, G.; Eisemann, L.; Michel, M.; Zell, S. Universe's Primordial Quantum Memories. J. Cosmol. Astropart. Phys. 2019, 03, 010. [CrossRef]

45. Dvali, G.; Gomez, C. Self-Completeness of Einstein Gravity. arXiv 2010, arXiv:1005.3497.

46. Dvali, G.; Giudice, G.F.; Gomez, C.; Kehagias, A. UV-Completion by Classicalization. J. High Energy Phys. 2011, 8, 108. [CrossRef] 